Supporting information:

\title{
Single-entity approach to investigate surface charge enhancement in magnetoelectric nanoparticles induced by $\mathrm{AC}$ magnetic field stimulation
}

Popular Pandey, ${ }^{1}$ Govinda Ghimire ${ }^{1}$, Javier Garcia ${ }^{1}$, Alberto Rubfiaro ${ }^{1}$, Xuewen Wang ${ }^{1}$, Asahi Tomitaka ${ }^{2}$, Madhavan Nair ${ }^{2}$, Ajeet Kaushik ${ }^{*}$, Jin He $\mathrm{He}^{1,4^{*}}$

${ }^{1}$ Physics Department, Florida International University, Miami, Florida, 33199, United States ${ }^{2}$ Department of Immunology and Nanomedicine, Institute of Neuroimmune Pharmacology, Herbert Wertheim College of Medicine, Florida International University, Miami, Florida, 33199, United States ${ }^{3}$ NanoBioTech Laboratory, Department of Natural Sciences, Division of Sciences, Art, \& mathematics, Florida Polytechnic University, Lakeland, Florida, 33805, United States

${ }^{4}$ Biomolecular Science Institute, Florida International University, Miami, Florida, 33199, United States 


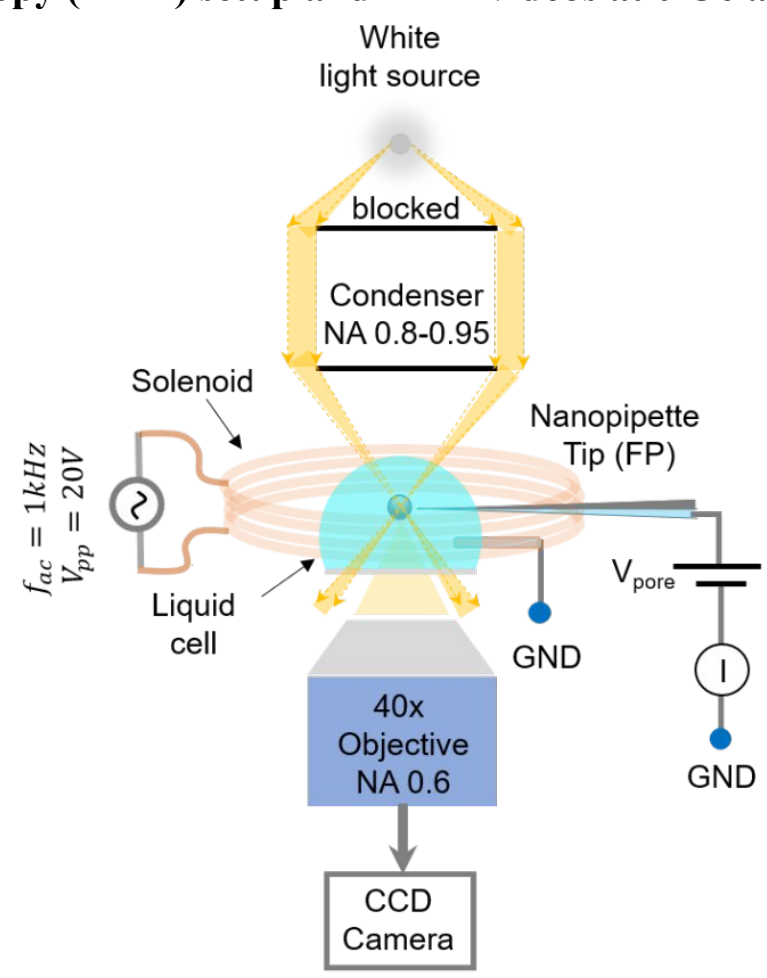

Figure S1. Schematic setup of the DFM. When the objective is focused at the nanopipette tip, tip serves as a focal point (FP).

S1a. DF video of MENP with and without AC B-field

S1b. DF video of MNP with and without AC B-field

By implementing the DF experimental setup shown in Figure S1, the motion of the MENP (video S1a) and MNP (video S1b) without (0 Oe) and with $(60 \mathrm{Oe}) \mathrm{AC}$ B-field were recorded and analyzed. The bright spots in the video are individual NPs and the elongated bright tip at the lower right corner of the video is the nanopore-CNE nanopipette tip. 


\section{S2. Theoretical estimation of AC B-field stimulated surface potential enhancement of MENP}

Here we consider the simplest case where the CFO core and BTO shell are tightly connected at the interface such that any stress generated at the CFO core can be completely transferred to the BTO shell. The solenoid generates the magnetic field along the $z$-axis. In a dielectric ceramic material like BTO without the center of symmetry, an electric polarization $(P)$ is induced due to the external mechanical stress $(\sigma)$.

$P=d * \sigma($ Direct effect $)$ and

$\varepsilon=d * E($ Converse effect)

Where $d$ is the piezoelectric coefficient $(\mathrm{m} / \mathrm{V})$ and $\varepsilon$ is the strain produced due to stress.

Assuming the field and the displacement along the $z$-direction, the value of $d$ for BTO is $200 \mathrm{pC} / \mathrm{N}$. Or $\mathrm{pm} / \mathrm{V} \cdot{ }^{1}$ The strain $(\varepsilon=\Delta l / l)$ produced in the BTO shell can be calculated by taking the ratio of change in dimension to the original dimension. The maximum change in the thickness of the BTO layer under $60 \mathrm{~Hz}$ of $\mathrm{AC}$ frequency and 50 Oe field is $\sim-43 \mathrm{ppm} .^{2}$ Note that $43 \mathrm{ppm}$ is $0.0043 \%$. Thus, $\Delta l$ is $0.0043 \%$ of the shell thickness $l(\sim 6 \mathrm{~nm})$. The AC magnetic field magnitude and frequency in our experiment is $\sim 60$ Oe and $1 \mathrm{kHz}$.

Thus, using $\varepsilon=\Delta l / l$ and $E=V / l$ in equation (2) gives

$\Delta l=d * V$.

Thus, potential V generated by the stress is approximated to be $-1.30 \mathrm{mV}$.

This is an estimation of the local potential generated on the surface of a single MENP under AC B-field stimulation. 


\section{S3. Characteristics of the nanopore-CNE nanopipettes used in the study}

Details of the fabrication and characterization of nanopore-CNE nanopipettes can be found in our previous publication..$^{3-5}$ The diameter of the nanopore ranges from 40-100 $\mathrm{nm}$. The CNE effective surface area ranges between $0.02-1.02 \mu \mathrm{m}^{2}$ with a mean value of $0.21 \mu \mathrm{m}^{2}$. The detailed information of each nanopipette is summarized in table $\mathrm{S} 1$.

Table S1. The nanopore diameter and the effective CNE surface area of 8 nanopipettes.

\begin{tabular}{|c|c|c|c|c|}
\hline Nanopipette & $\begin{array}{c}\text { CNE Area } \\
\left(\boldsymbol{\mu m}^{2}\right)^{\mathrm{a}}\end{array}$ & $\begin{array}{c}\text { CNE radius } \\
(\mathbf{n m})\end{array}$ & $\begin{array}{c}\text { Nanopore } \\
\text { diameter }(\mathbf{n m})^{\mathrm{b}}\end{array}$ & $\begin{array}{c}\text { Measurement } \\
\mathbf{s}\end{array}$ \\
\hline P1 & $0.378 \pm 0.026$ & $241 \pm 64$ & $96 \pm 9$ & MENP \\
\hline P2 & $0.091 \pm 0.006$ & $120 \pm 31$ & $95 \pm 9$ & MNP \\
\hline P3 & $0.078 \pm 0.005$ & $111 \pm 29$ & $58 \pm 5$ & MENP \\
\hline P4 & $0.488 \pm 0.034$ & $278 \pm 73$ & $71 \pm 6$ & MNP \\
\hline P5 & $0.080 \pm 0.005$ & $113 \pm 29$ & $60 \pm 5$ & MENP \\
\hline P6 & $0.094 \pm 0.007$ & $120 \pm 37$ & $138 \pm 13$ & MNP \\
\hline P7* & $0.344 \pm 0.024$ & $234 \pm 61$ & $85 \pm 8$ & MENP \\
\hline P8 & $0.017 \pm 0.001$ & $89 \pm 24$ & $44 \pm 4$ & MENP \\
\hline
\end{tabular}

aThe error in the CNE effective area is mainly due to the uncertainly of geometry. ${ }^{b}$ The error in the nanopore diameter is calculated based on the uncertainty of the nanopipette geometry (half cone angle). *chemically modified nanopipette. 


\section{S4. Estimation of the forces acting on the motion of MENP and MNP under a AC B-field.}

There are three major forces affecting the motion of NPs in our single-NP experiments: (i) Electrical force $\left(\mathbf{F}_{\mathbf{e}}\right)$ due to the charge of the nanoparticle; (ii) Magnetic force $\left(\mathbf{F}_{\mathbf{m}}\right)$ due to the $\mathrm{AC}$ B-field and (iii) Stoke's drag force $\left(\mathbf{F}_{\mathbf{d}}\right)$ due to the viscosity of the medium. Additionally, there is also torque associated with the magnetic field and the fluid friction. The parameters required to estimate these forces are presented in the table $\mathrm{S} 2$ below.

Table.S2 The parameters used in the calculations

\begin{tabular}{|c|c|c|c|c|c|c|c|c|}
\hline Parameters & $\begin{array}{l}\text { Diameter } \\
\qquad \begin{array}{l}\mathrm{d} \\
\text { (m) }\end{array}\end{array}$ & $\begin{array}{l}\text { Surface } \\
\text { charge } \\
\text { density } \sigma^{\text {a }} \\
\left(\mathrm{C} / \mathrm{m}^{2}\right)\end{array}$ & $\begin{array}{c}\text { Electric } \\
\text { Field } \\
\text { E } \\
(\mathrm{V} / \mathrm{m})^{\mathrm{b}}\end{array}$ & $\begin{array}{c}\text { Viscosity } \\
\text { of water } \\
\eta \\
(\mathrm{kg} / \mathrm{m} . \mathrm{s})\end{array}$ & $\begin{array}{l}\text { Magnetic } \\
\text { moment } \\
m=M \rho V \\
\left(\mathrm{Am}^{2}\right)\end{array}$ & $\begin{array}{c}\text { Specific } \\
\text { magnetization } \\
(M) \\
\left(\mathrm{Am}^{2} / \mathrm{kg}\right)\end{array}$ & $\begin{array}{c}\text { Density } \\
\rho \\
\left(\mathrm{kg} / \mathrm{m}^{3}\right)\end{array}$ & $\begin{array}{c}\text { Magnetic Field } \\
\qquad B \\
\text { (T) }\end{array}$ \\
\hline MENP & $25 \times 10^{-9}$ & $23 \times 10^{-3}$ & $10^{7}$ & $1.0 \times 10^{-3}$ & $1.5 \times 10^{-18}$ & $34^{\mathrm{c}}$ & $5299^{c}$ & $6 \times 10^{-3}$ \\
\hline MNP & $50 \times 10^{-9}$ & $16^{6} \times 10^{-3}$ & $10^{7}$ & $1.0 \times 10^{-3}$ & $9.5 \times 10^{-17}$ & $80^{\mathrm{d}}$ & $5400^{\mathrm{d}}$ & $6 \times 10^{-3}$ \\
\hline
\end{tabular}

Note: ${ }^{\text {TThe }}$ surface charge densities of MENP and MNP are estimated from zeta potential mesurements. ${ }^{b}$ The $\mathrm{E}$ at the nanopipette apex is adopted from the simulation of previous work. ${ }^{5}$ ${ }^{\mathrm{c}}$ The density and specific magnetization of MENP are taken from previous work. ${ }^{6-8}$ ${ }^{\mathrm{d}}$ The density and specific magnetization of MNP are taken from previous report. ${ }^{8}$

Table S3: Force $(F)$, torque $(\tau)$ and MENP/MNP rotational parameters calculations

\begin{tabular}{|c|c|c|}
\hline Electric force $\left(\mathrm{F}_{\mathrm{e}}\right)$ & $\mathrm{F}_{\mathrm{e}}=\mathrm{q} * \mathrm{E}=\frac{\pi}{4} \sigma d^{2} E$ & $\sim 10^{-10} \mathrm{~N}$ \\
\hline Magnetic Force $\left(\mathrm{F}_{\mathrm{m}}\right)$ & $\mathrm{F}_{\mathrm{m}}=(m . \nabla) B=\rho * V(M . \nabla) B$ & zero at the uniform B-field \\
\hline Magnetic torque $\left(\tau_{\mathrm{m}}\right)^{9,10}$ & $\begin{array}{c}\tau_{\mathrm{m}}=m \times B=M V * B \\
\tau_{m}=\frac{V \chi^{2} B^{2} \operatorname{Sin}(2 \theta)}{2(2+\chi)} \\
2 \theta=15^{0} \text { (assume) and } \\
\chi=2 \text { for } M E N P \text { and } 5 \text { for } M_{N P}^{9}\end{array}$ & $\begin{array}{l}\text { MENP: } 0.01 p N \mathrm{~nm} \\
\text { MNP: } 0.08 p N \mathrm{~nm}\end{array}$ \\
\hline Frictional torque $\left(\tau_{\mathrm{f}}\right)^{10,11}$ & $\tau_{\mathrm{f}}=8 \pi \eta r^{3} \omega$ & $\begin{array}{l}30 \mathrm{~nm} \text { MENP: } 7.55 \times 10^{-26} \omega \mathrm{Nm} \\
50 \mathrm{~nm} \text { MNP: } 3.49 \times 10^{-25} \omega \mathrm{Nm}\end{array}$ \\
\hline Angular velocity $(\omega)$ & $\omega=\frac{V \chi B^{2} \operatorname{Sin}(2 \theta)}{2 \mu_{0}(2+\chi) 8 \pi \eta r^{3}}$ & $\begin{array}{l}30 \mathrm{~nm} \text { MENP: } 433 \mathrm{rad} / \mathrm{s} \\
50 \mathrm{~nm} \mathrm{MNP:} 1032 \mathrm{rad} / \mathrm{s}\end{array}$ \\
\hline $\begin{array}{c}\text { Tangential velocity }(v) \text { at } \\
\text { the surface }\end{array}$ & $v=\omega r$ & $\begin{array}{l}30 \mathrm{~nm} \text { MENP: } 6.5 \mu \mathrm{m} / \mathrm{s} \\
50 \mathrm{~nm} \text { MNP: } 25.8 \mu \mathrm{m} / \mathrm{s}\end{array}$ \\
\hline Frequency of rotation $(f)$ & $f=\omega / 2 \pi$ & $\begin{array}{l}30 \mathrm{~nm} \text { MENP: } 69 \mathrm{~Hz} \\
50 \mathrm{~nm} \text { MNP: } 164 \mathrm{~Hz}\end{array}$ \\
\hline Stoke's drag Force $\left(\mathrm{F}_{\mathrm{d}}\right)$ & $\mathrm{F}_{\mathrm{d}}=3 \pi * \eta * d * v ; v \sim 10^{-6} \mathrm{~m} / \mathrm{s}$ & $\sim 10^{-15} \mathrm{~N}$ \\
\hline
\end{tabular}


The main force in the experiment is $\mathrm{F}_{\mathrm{e}}$. The drag force is comparable to the force due to Brownian motion. Since the externally applied magnetic field is uniform, there is no magnetic force applied on the magnetic NPs. However, there is a magnetic torque $\left(\tau_{\mathrm{m}}\right)$ applied on the NPs, which cause the rotational motion. We estimated that the magnetic torque applied on the MENP in presence of $6 \mathrm{mT}$ AC B-field is an order magnitude small than that on the MNP. As the NP rotates, it also experiences the frictional torque. In Newtonian fluids, the frictional torque $\left(\tau_{\mathrm{f}}\right)$ for rotation can be expressed as $8 \pi \eta r^{3} \omega$, where $\omega$ is the angular velocity of the nanoparticle and $\eta$ is the fluid viscosity. In the inertia-less limit, the frictional torque balances with magnetic torque. This allows us to estimate the terminal angular velocity of the magnetic NP. 


\section{S5. MENP and MNP translocation events}

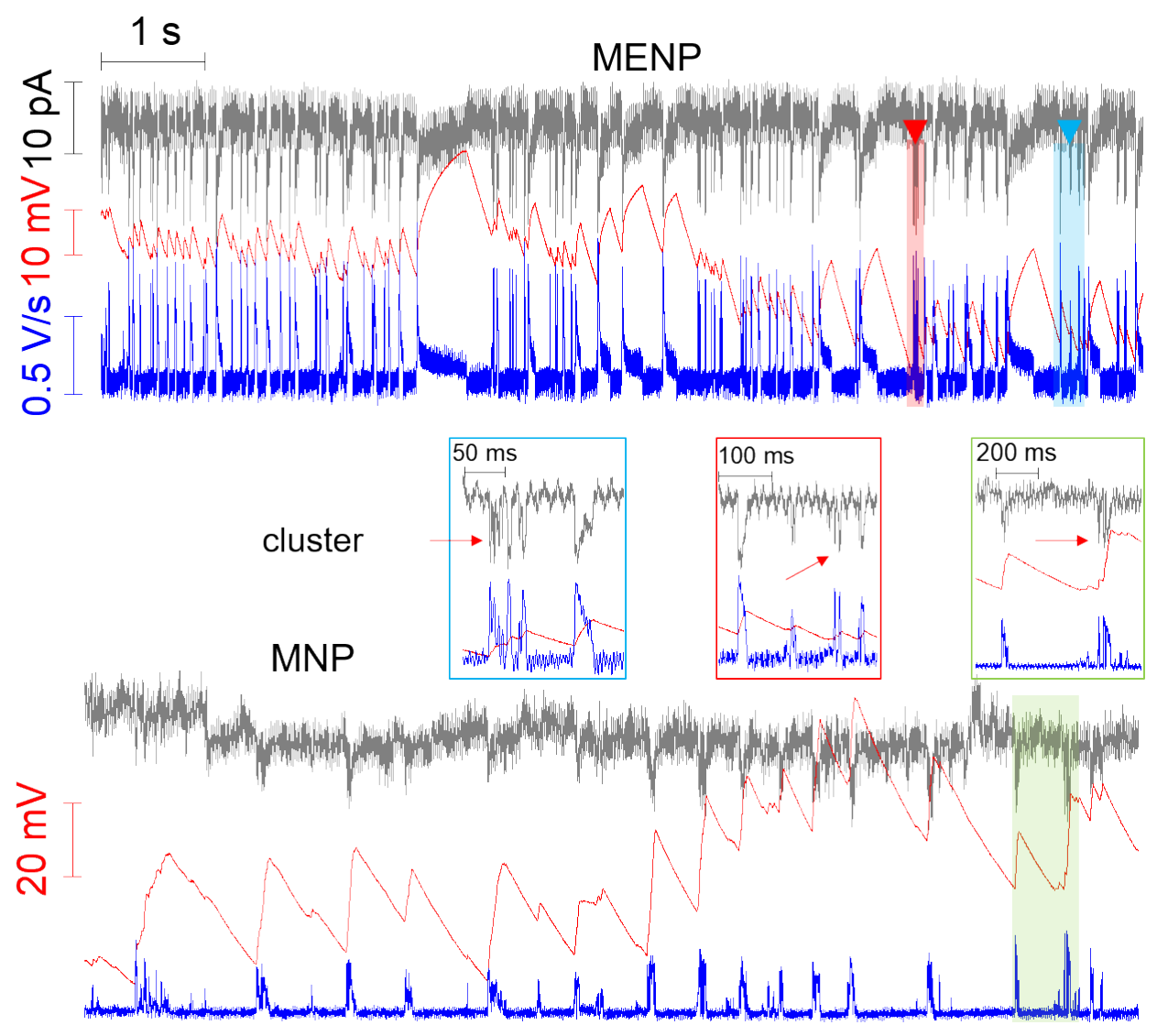

Figure S2. Typical time traces of current (gray), potential (red) and potential first derivative (blue) for the MENP (top panel) and MNP (bottom panel) nanopore translocation events at 60 Oe $\left(\mathrm{V}_{\text {pore }}=400 \mathrm{mV}\right)$ AC B-field. Nanopipettes P5 and P6 were used to obtain the MENP and MNP translocation data, respectively. Occasionally, clustered events (highlighted in the zoomed-in windows) were observed in both MENP and MNP experiments. The red arrows denote the clustered translocation events. The $\mathrm{dV} / \mathrm{dt}$ traces were smoothed by the moving average method using a $2 \mathrm{~ms}$ time window. 
S6. Extra experimental data of single MENP and MNP collision events at the CNE without/with AC B-field

\section{S6a: MENP-CNE collision events}

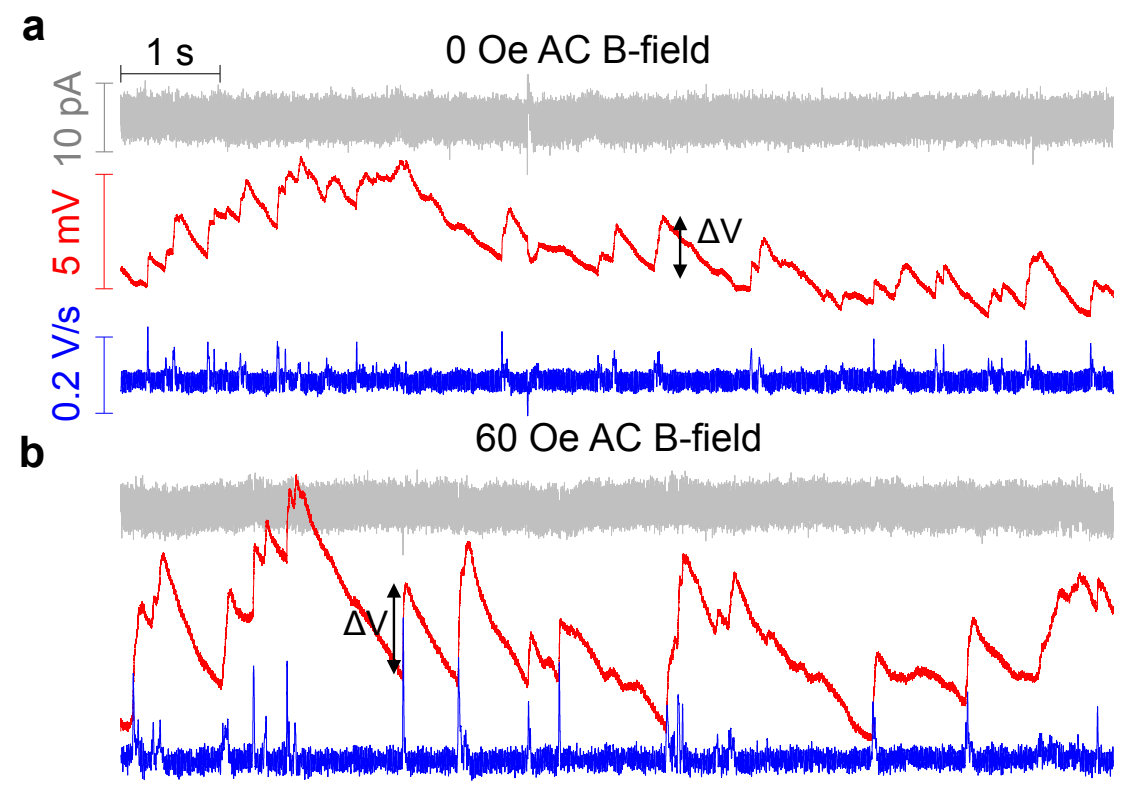

Figure S3a. The time traces of current (black), potential (red), and the first derivative of potential (blue) of MENP collision to the CNE at $\mathrm{V}_{\text {pore }}=400 \mathrm{mV}$ (a) 0 Oe AC B-field (b) 60 Oe AC B-field using nanopipette $\mathrm{P} 1$. The $\mathrm{dV} / \mathrm{dt}$ curves were smoothed by the moving average method using a 2 ms time window. 


\section{S6b: MNP-CNE collision events}

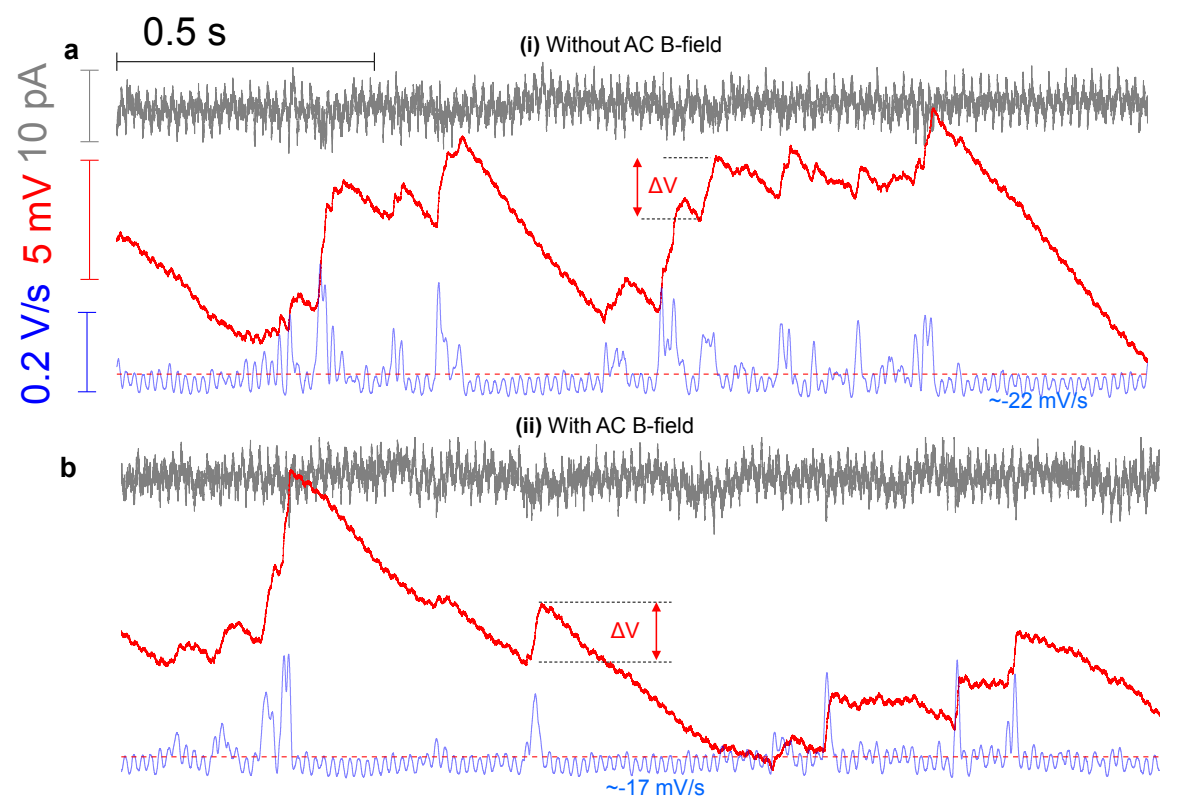

Figure S3b. The time traces of current (gray), potential (red) and the first derivative of potential (blue) of MNP collision events at the CNE at $\mathrm{V}_{\text {pore }}=400 \mathrm{mV}$ (a) without (b) with AC $\mathrm{B}$-field. The nanopipette $\mathrm{P} 2$ is used to acquire the data and the concentration of MNP in 10 $\mathrm{mM}$ PBS is $1 \mathrm{nM}$. The $\mathrm{dV} / \mathrm{dt}$ traces were smoothed by the moving average method using a 10 ms time window. 


\section{S6c: Single vs. cluster MENP-CNE collision events}
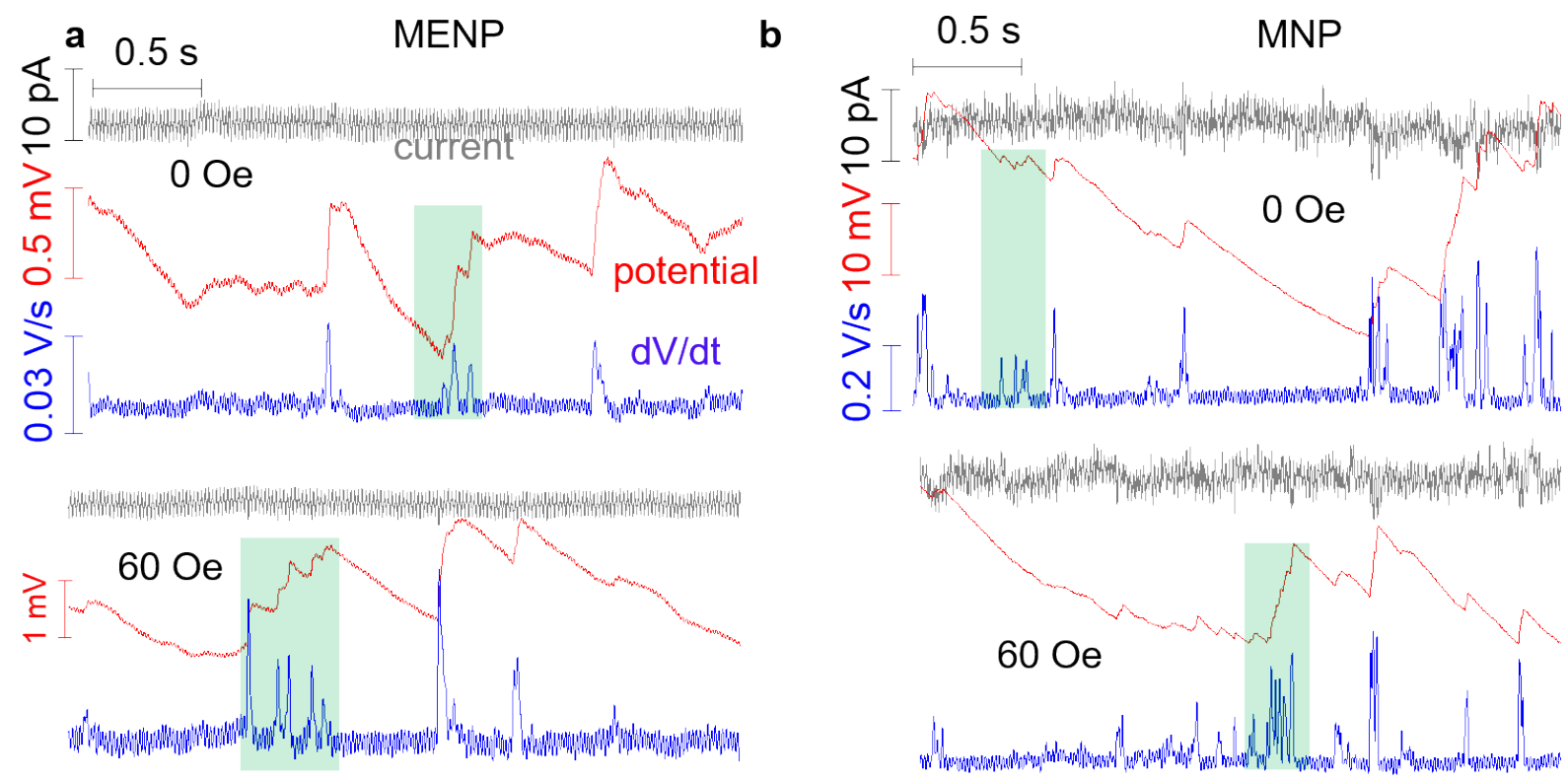

Figure S3c. The time traces of current (gray), potential (red) and the first derivative of potential (blue) of (a) MENP-CNE and (b) MNP-CNE collision events at 0 Oe and 60 Oe AC B-field. The highlighted regions denote the collisions events due to a nanoparticle cluster. By analyzing the shape of potential and $\mathrm{dV} / \mathrm{dt}$ time traces, the single nanoparticle collision events were differentiated. We have only analyzed the isolated collision spikes unaffected by adjoining nanoparticles. The nanopipettes P1and P2 were used to acquire MENP and MNP experimental data and the concentration of the nanoparticles in $10 \mathrm{mM}$ PBS is $1 \mathrm{nM}$. The $\mathrm{dV} / \mathrm{dt}$ traces were smoothed by the moving average method using a $10 \mathrm{~ms}$ time window. 


\section{S7: The event rate of MNP-CNE collision event without/with a AC B-field}

The event rate (/s) as a function of time for MNP-CNE collision at the CNE without and with the presence of AC B-field is shown in Figure S6. Same as Figure $2 b$ in the main text, there are apparent fluctuations in the event rate at both zero and $60 \mathrm{Oe} \mathrm{AC}$ B-field. These fluctuations of the collision event rate are attributed to the fluctuations of the local NP concentration. The average rate is about 0.25 events $/ \mathrm{s}$ at zero Oe and about 0.22 events $/ \mathrm{s}$ at $60 \mathrm{Oe}$. The overall average event rate is $\sim 0.24$ events/s. At $60 \mathrm{Oe}$, the MNPs are subjected to both translocational and rotational motions (see $\mathrm{S} 4$ of SI), which may slightly reduce the event rate.

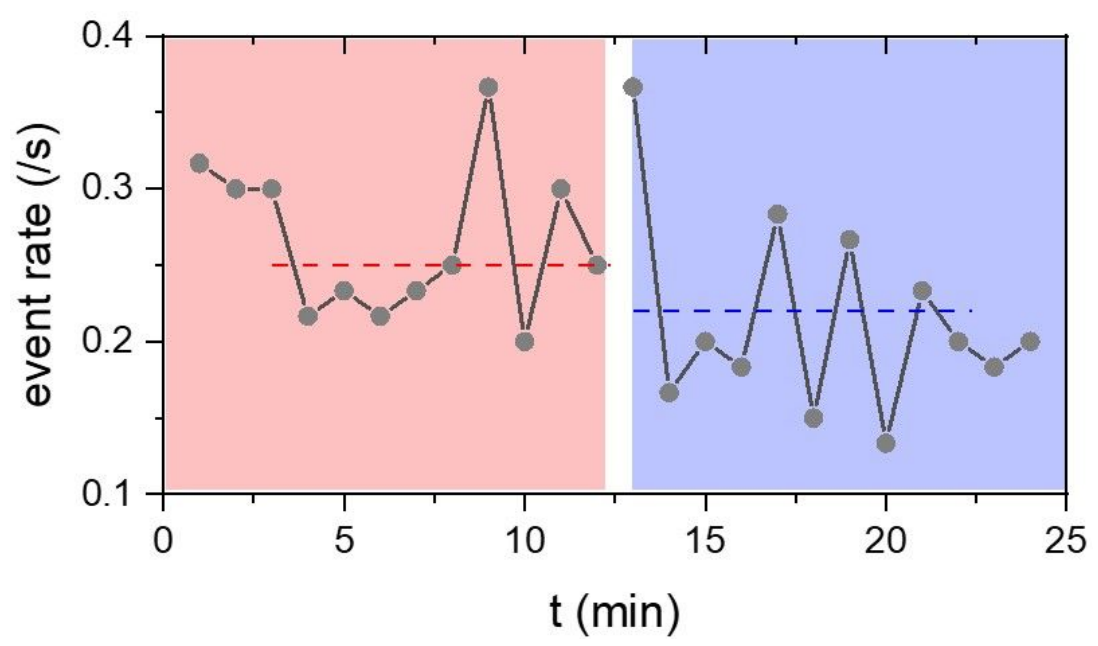

Figure S4. The event rate of MNP-CNE collision event without (light red region) and with a 60 Oe (light blue region) AC B-field as a function of time. The average event rates over 10 minutes at 0 and $60 \mathrm{Oe}$ AC B-field are 0.25 and 0.22 events/s, respectively. The nanopipette $\mathrm{P} 2$ was used to acquire the data at $400 \mathrm{mV}$ nanopore bias. The concentration of MNP in 10 $\mathrm{mM}$ PBS was $1 \mathrm{nM}$. 


\section{S8: Diffusion-limited event rates based on Stokes-Einstein relationship}

Stokes-Einstein relationship for the diffusion coefficient $D$ of a spherical particle of radius $r$ in a fluid of dynamic viscosity $\eta$ at absolute temperature $T(\mathrm{~K})$ is given as

$$
D=\frac{k_{B} T}{6 \pi \eta r^{\prime}}
$$

where, $k_{B}\left(=1.3803 \times 10^{-23} \mathrm{~J} \mathrm{~K}^{-1}\right)$ is the Boltzmann's constant and $\eta\left(=1.0 \times 10^{-3} \mathrm{Kg} / \mathrm{ms}\right)$ is the viscosity of water.

If we only consider the diffusive motion of the particles to the hemispherical electrode, the frequency of the particle collision event at the electrode, $f_{\text {diff }}$, can be estimated using the following equation,

$$
f_{\text {diff }}=4 D C_{N P} r_{C N E} N_{A},
$$

where $D$ is the diffusion coefficient of the NPs, $C_{N P}$ is the bulk concentration of the NP in particles/ $m^{3}, r_{C N E}$ is the nanoelectrode radius and $N_{A}$ is Avogadro's number. To compare with the experiments, we used the $\mathrm{CNE}$ radius of nanopipettes P1 and P6. The radii of P1 and P6 are 241 $\pm 64 \mathrm{~nm}$ and $120 \pm 37 \mathrm{~nm}$, respectively. The calculated and experimental (with $\mathrm{V}_{\text {pore }}=+400 \mathrm{mV}$ ) results of $\mathrm{P} 1$ and $\mathrm{P} 6$ are both shown in table $\mathrm{S} 4$ below. In the experiment using zero bias and without AC B-field, the mean MENP-CNE and MNP-CNE collision event rate was $0.46 / \mathrm{s}$ and $0.21 / \mathrm{s}$ respectively.

Table S4. Theoretical and experimental MENP-CNE and MNP-CNE collision events frequency $(/ \mathrm{s})$

\begin{tabular}{|c|c|c|c|c|c|}
\hline & \multicolumn{2}{|c|}{ Theory } & \multicolumn{2}{c|}{ Experiment $\mathrm{V}_{\text {pore }}=400 \mathrm{mV}$} & Experiment $\mathrm{V}_{\text {pore }}=0 \mathrm{mV}$ \\
\hline & $\begin{array}{c}D \times \\
10^{-7} \\
\left(\mathrm{~cm}^{2} / \mathrm{s}\right)\end{array}$ & $f_{\text {diff }}(/ \mathrm{s})$ & $f_{\text {diff }} @ 0$ Oe & $f_{\text {diff }} @ 60 \mathrm{Oe}$ & $f_{\text {diff }} @ 0$ Oe \\
\hline MENP & 1.46 & 8.45 & 1.30 & 1.27 & 0.46 \\
\hline MNP & 0.44 & 1.26 & 0.25 & 0.22 & 0.21 \\
\hline
\end{tabular}

Table S5. The event rate (events/s) of MENP-CNE collision events from theory and experiment.

\begin{tabular}{|c|c|c|c|c|c|c|}
\hline & \multicolumn{2}{|c|}{ Theory } & \multicolumn{2}{c|}{ Experiment $\mathrm{V}_{\text {pore }}=0 \mathrm{mV}$} & \multicolumn{2}{c|}{ Experiment $\mathrm{V}_{\text {pore }}=400 \mathrm{mV}$} \\
\hline & $\begin{array}{c}D \times 10^{-7} \\
\left(\mathrm{~cm}^{2} / \mathrm{s}\right)\end{array}$ & $f_{\text {diff }}(/ \mathrm{s})$ & $f_{\text {diff }} @ 0 \mathrm{Oe}$ & $f_{\text {diff }} @ 60 \mathrm{Oe}$ & $f_{\text {diff }} @ 0 \mathrm{Oe}$ & $f_{\text {diff }} @ 60 \mathrm{Oe}$ \\
\hline MENP & 1.46 & 8.45 & 0.26 & 0.16 & 0.33 & 0.18 \\
\hline
\end{tabular}

The nanopipette P8 was used to perform this experiment. 
S9: The $\triangle \mathrm{V}$ distributions of MENP and MNP collision events at different AC B-field magnitude
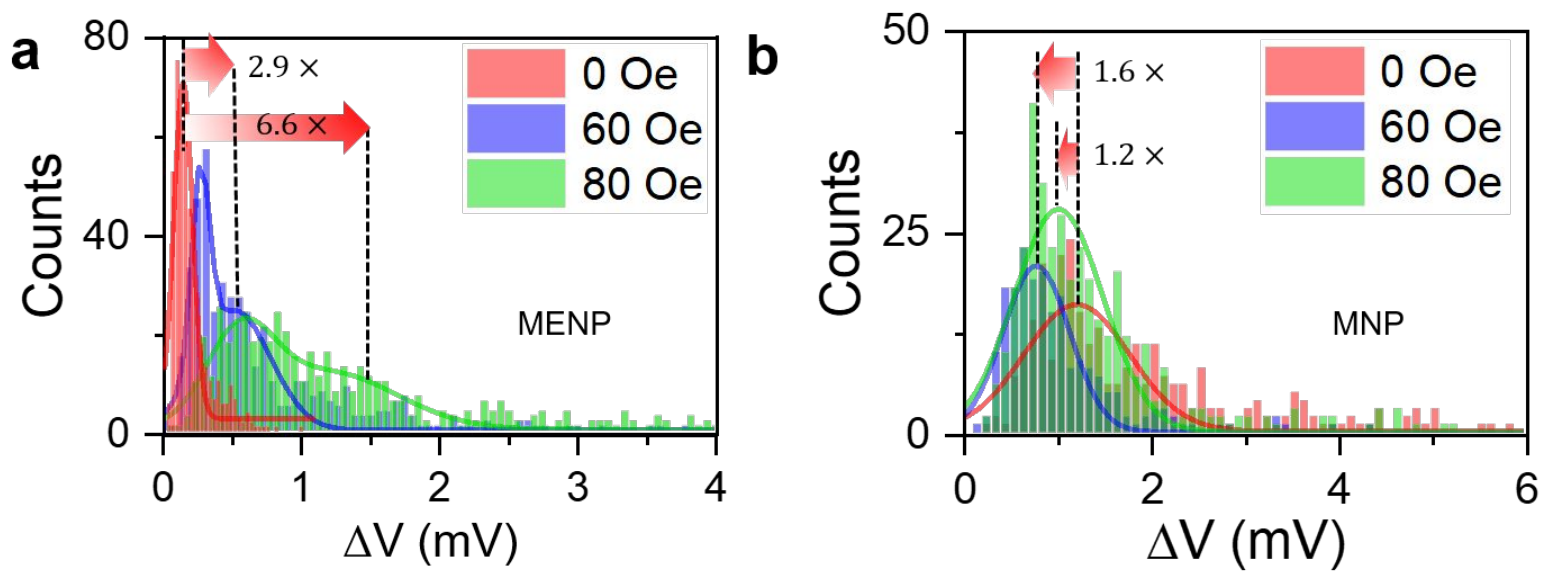

Figure S5. The changes of $\Delta \mathrm{V}$ as a function of the AC B-field magnitude. (a) The histograms of $\Delta \mathrm{V}$ of the potential dip of the MENP-CNE collision events at 0,60 and 80 Oe. The most probable values are $0.18 \pm 0.07,0.52 \pm 0.26$ and $1.19 \pm 0.50 \mathrm{mV}$, respectively. (b) The histograms of the $\Delta \mathrm{V}$ of the potential dip of MNP-CNE collision events at 0,60 and 80 Oe. The most probable values are $1.22 \pm 0.56,0.78 \pm 0.35$ and $1.02 \pm 0.47 \mathrm{mV}$, respectively. The solid lines in the histograms are the Gaussian fits. 


\section{S10: The effect of the applied nanopore bias $\left(V_{\text {pore }}\right)$ on the MENP-CNE collision events}

The Potential dip amplitude is almost the same at $0 \mathrm{mV}$ and $400 \mathrm{mV}$ nanopore bias when no magnetic field is present. The potential dip amplitude increased by $\sim 34 \%$ at $400 \mathrm{mV}$ nanopore bias compared to $0 \mathrm{mV}$ when $\mathrm{AC} \mathrm{B}$-field is present. The increased potential amplitude is due to the increased surface charge of the MENP and larger electrophoretic force.
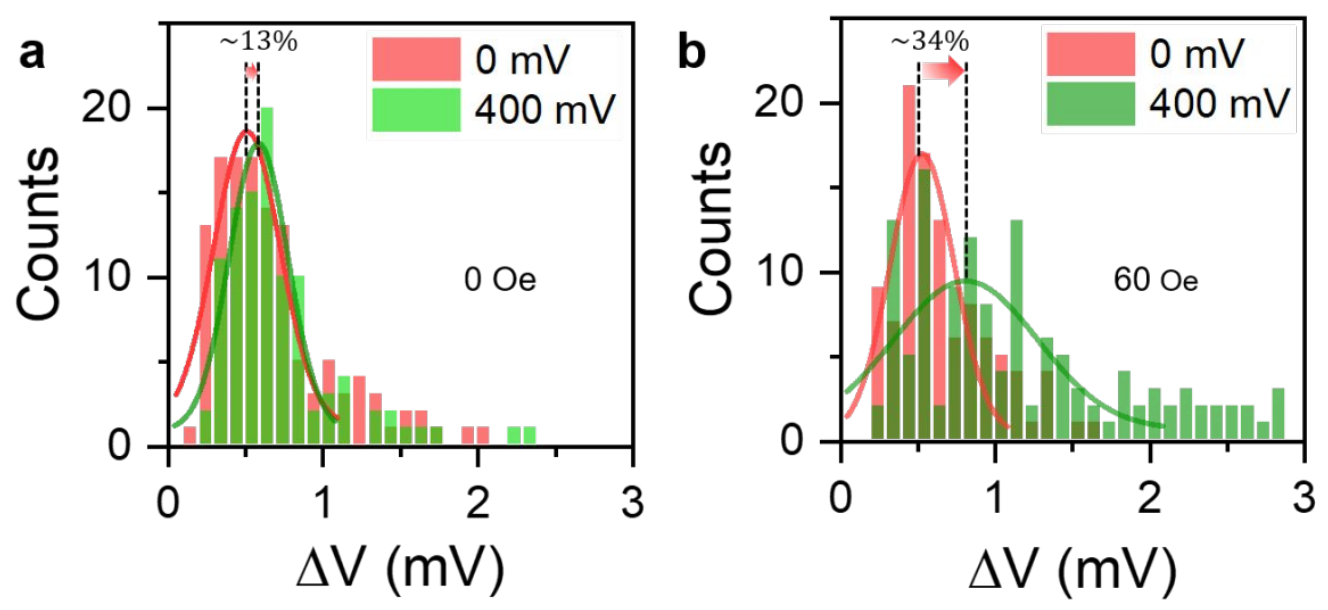

Figure S6. (a) Effect of the nanopore bias $\left(\mathrm{V}_{\text {pore }}\right)$ on the potential dip amplitude $\Delta \mathrm{V}$ during the MENP-CNE collision without (a) and with 60 Oe (b) AC B-field using the nanopipette P8. The estimated radius of the $\mathrm{CNE}$ is about $89 \pm 24 \mathrm{~nm}$. The most probable values of $\Delta \mathrm{V}$ for MENPCNE collision events at $0 \mathrm{mV}$ and $400 \mathrm{mV}$ nanopore biases are $0.51 \pm 0.21 \mathrm{mV}$ and $0.59 \pm$ $0.19 \mathrm{mV}$ respectively. The most probable values of $\Delta \mathrm{V}$ for MNP-CNE collision events at $0 \mathrm{mV}$ and $400 \mathrm{mV}$ nanopore biases are $0.54 \pm 0.21 \mathrm{mV}$ and $0.82 \pm 0.47 \mathrm{mV}$ respectively. The solid lines in the histograms are the Gaussian fits. 


\section{S11: MENP-CNE collision events using a chemically modified nanopipette}

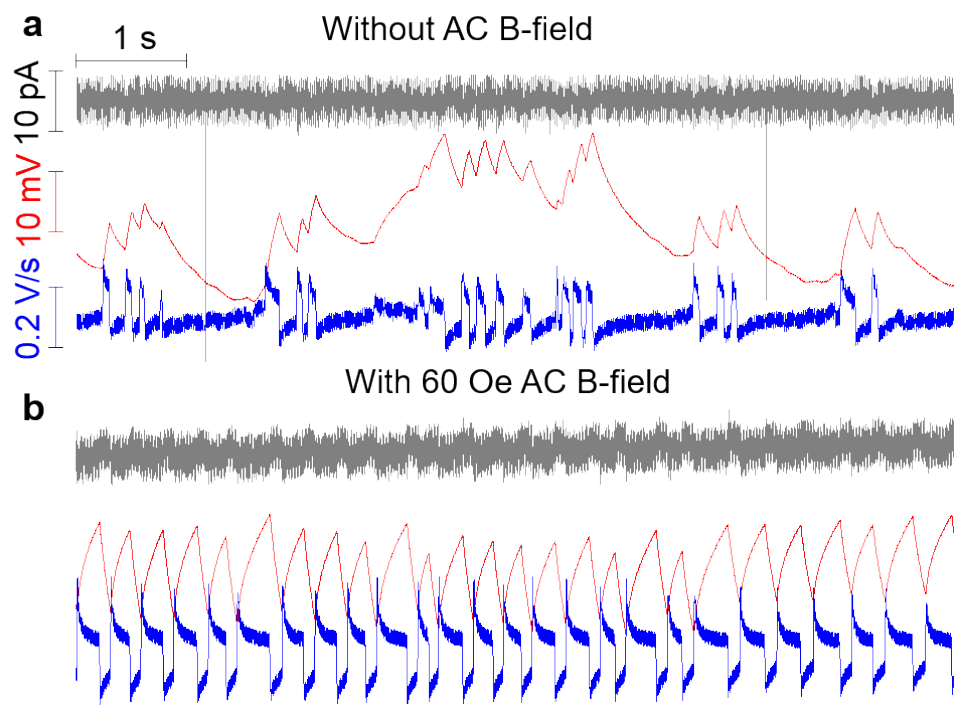

Figure S7. The time traces of current (grey), potential (red) and the first derivative of potential (blue) of MENP collision events at the CNE at (a) 0 Oe and (b) 60 Oe AC B-field. The nanopore bias $\mathrm{V}_{\text {pore }}=400 \mathrm{mV}$. The nanopipette $\mathrm{P} 7$ is used to acquire the data and the concentration of MENP is $1 \mathrm{nM}$ in $10 \mathrm{mM}$ PBS. The $\mathrm{dV} / \mathrm{dt}$ trace was smoothed using the moving average method using a $10 \mathrm{~ms}$ time window.

Because of the reduced electrostatic repulsing force, the NPs tend to form loosely coupled clusters at the zero AC B-field. These clustered potential dips can still be separated from each other, which is clearly demonstrated in the $\mathrm{dV} / \mathrm{dt}$ trace. 


\section{REFERENCES}

1. Dong, L.; Stone, D. S.; Lakes, R. S., Enhanced dielectric and piezoelectric properties of xBaZrO3(1-x)BaTiO3 ceramics. Journal of Applied Physics 2012, 111 (8), 084107.

2. Betal, S.; Shrestha, B.; Dutta, M.; Cotica, L. F.; Khachatryan, E.; Nash, K.; Tang, L.; Bhalla, A. S.; Guo, R., Magneto-elasto-electroporation (MEEP): In-vitro visualization and numerical characteristics. Scientific Reports 2016, 6, 32019.

3. Pandey, P.; Garcia, J.; Guo, J.; Wang, X.; Yang, D.; He, J., Differentiation of metallic and dielectric nanoparticles in solution by single-nanoparticle collision events at the nanoelectrode. Nanotechnology 2019, 31 (1), 015503.

4. Pandey, P.; Panday, N.; Chang, S.; Pang, P.; Garcia, J.; Wang, X.; Fu, Q.; He, J., Probing Dynamic Events of Dielectric Nanoparticles by a Nanoelectrode-Nanopore Nanopipette. ChemElectroChem 2018, 5 (20), 3102-3112.

5. Panday, N.; Qian, G.; Wang, X.; Chang, S.; Pandey, P.; He, J., Simultaneous lonic Current and Potential Detection of Nanoparticles by a Multifunctional Nanopipette. ACS Nano 2016, 10 (12), 1123711248.

6. $\quad$ Kaushik, A.; Nikkhah-Moshaie, R.; Sinha, R.; Bhardwaj, V.; Atluri, V.; Jayant, R. D.; Yndart, A.; Kateb, B.; Pala, N.; Nair, M., Investigation of ac-magnetic field stimulated nanoelectroporation of magneto-electric nano-drug-carrier inside CNS cells. Scientific Reports 2017, 7, 45663.

7. Rafferty, A.; Prescott, T.; Brabazon, D., Sintering behaviour of cobalt ferrite ceramic. Ceramics International 2008, 34 (1), 15-21.

8. Golovin, Y. I.; Klyachko, N. L.; Majouga, A. G.; Sokolsky, M.; Kabanov, A. V., Theranostic multimodal potential of magnetic nanoparticles actuated by non-heating low frequency magnetic field in the new-generation nanomedicine. Journal of Nanoparticle Research 2017, 19 (2), 63.

9. Gabayno, J. L. F.; Liu, D.-W.; Chang, M.; Lin, Y.-H., Controlled manipulation of Fe3O4 nanoparticles in an oscillating magnetic field for fast ablation of microchannel occlusion. Nanoscale 2015, 7 (9), 39473953.

10. Moerland, C. P.; van Ijzendoorn, L. J.; Prins, M. W. J., Rotating magnetic particles for lab-on-chip applications - a comprehensive review. Lab on a Chip 2019, 19 (6), 919-933.

11. Janssen, X. J. A.; Schellekens, A. J.; van Ommering, K.; van ljzendoorn, L. J.; Prins, M. W. J., Controlled torque on superparamagnetic beads for functional biosensors. Biosensors and Bioelectronics 2009, 24 (7), 1937-1941. 